\title{
DIVERSAS CONEXIONES ENTRE CELESTINA Y ELICIA ${ }^{1}$
}

\author{
José Antonio Giménez Micó \\ Universidad de Montreal
}

\begin{abstract}
Quizá la diferencia fundamental entre un texto literario y otro que no lo es-Celestina y un tratado de mineralogía, por ejemplo-radica en su carácter connotativo, frente a la pretensión denotativa del segundo. Porque la obra literaria es, más que cualquier otra manifestación lingüística, imperfecta, en el sentido de que no está acabada. Cada una de las informaciones que ofrece guarda estrecha relación con algo que no aparece explícitamente, algo que el lector debe completar. ${ }^{2} Y$, lo que es más importante: cada lector lo completará diferentemente, de acuerdo a su competencia, a su ideología, a su axiología; a las evocaciones que en él despierte. Lo que en un discurso médico, jurídico o histórico tiende a ofrecer un aspecto unívoco, se multiplica en el discurso literario. Éste es
\end{abstract}

${ }^{1}$ Una primera versión de este trabajo fue presentada en el congreso de la Asociación Canadiense de Hispanistas de 1992 (University of Prince Edward Island). El autor agradece a Félix Carrasco por sus valiosos consejos de orden teórico y metodológico. También agradece al FCAR ("Fonds pour la formation de chercheurs et l'aide à la recherche du Québec") y al SSHRCC ("Social Sciences and Humanities Research Council of Canada") por su apoyo financiero.

2 «En realidad todo enunciado es incompleto (lo mismo en la comunicación lingüística ordinaria que en la comunicación literaria) por presuponer más informaciones de las que da; porque cada información explícita en un entunciado no es sino una como tecla que pone en movimiento todo un contexto, todo un mundo en que se integra como fragmento de un todo mucho mayor y complejo, el de las representaciones y recuerdos que es capaz de evocar en el sujeto al que se dirige.» Antonio Gómez-Moriana. "La evocación como procedimiento en el Quijote," en Revista canadiense de estudios hispánicos 6 (invierno 1982), p. 194. 
polisémico, mientras que aquéllos son (o pretenden ser) monosémicos. De ahí las múltiples lecturas que pueden realizarse de toda obra literaria.

Múltiples, pero no infinitas. El modelo heurístico de obra $a b i e r t a^{3}$ es válido sólo relativamente, pues «un texte littéraire peut entraîner une série d'interprétations même différentes, mais quand même compatibles. ${ }^{4}$ Esta compatibilidad entre las diversas lecturas se debe a que tanto la producción como la recepción ${ }^{5}$ de toda obra se ven constreñidas por (y son posibles gracias a) una serie de códigos que se pueden respetar o no (ironía, parodia), pero nunca ignorar. En el análisis de un texto dado, la tarea del crítico ha de ser, sobre todo cuando se enfrenta "con una obra literaria del pasado, [...] la de reconstruir los códigos para arrancar las capas de sentido investidas en el texto," mediante la recuperación del "contexto socio-histórico de la comunicación artística.. ${ }^{6}$ Para ello es imprescindible analizar las interconexiones, no siempre armónicas, que se producen entre los diversos elementos del texto, y entre éste y el resto de obras pertenecientes a su área cultural, anteriores y contemporáneas; y también, entre el campo literario y los campos científico, filosófico, religioso, etc., vigentes en el contexto de producción del texto, o en cualquiera de sus contextos de recepción.

${ }^{3}$ «L'apertura, intesa come ambiguità fondamentale del messagio artistico.» Umberto Eco, Opera aperta, $2^{a}$ edición revisada y ampliada. Milán: Bompiani, 1967, p. 9.

${ }^{4}$ Hans Robert Jauss. Pour une herméneutique littéraire. Traducido del alemán al francés por Claude Maillard. Prólogo de Jean Starobinski. [Paris]: Gallimard, 1988, p. 367.

${ }^{5}$ Nos referimos aquí a la recepción competente, entendiendo esta palabra no en el sentido restringido de la gramática generativa, sino en el más lato que le otorga la crítica literaria desde hace algunos años ("la compétence est la connaissance par le lecteur du code linguistique, mais aussi des codes, conventions, contraintes sociales, des règles immanentes de lisibilité particulières au texte." Marc Angenot, Glossaire pratique de la critique contemporaine. LaSalle: Hurtubise, 1979, p. 49).

6 Félix Carrasco. "Notas a una lectura de 'Celestina' del siglo XVI: 'La comedia de Sepúlveda'," en Celestinesca 13.1 (mayo 1989), p. 43. 
Para poner en práctica las observaciones anteriores, compararemos Celestina ${ }^{7}$ con la Tragicomedia de Leandro y Roselia llamada Elicia, y por otro nombre cuarta obra y tercera Celestina, ${ }^{8}$ título que ya indica claramente que esta obra se incluye deliberadamente en lo que se ha venido en llamar "el ciclo celestinesco." Nuestro método estribará en analizar, en primer lugar, ciertos elementos formales y temáticos del texto primero; a continuación, compararemos estos elementos con sus correlatos del texto segundo, con el fin de intentar desentrañar la función de unos y otros en la producción de sentido.

Uno de los elementos de A que será retomado en B (como tendrá ocasión de comprobarse más tarde) es el monólogo que Pármeno pronuncia al final del segundo auto :

PARM.-[...] Pues anda que a mi cargo, ique Celestina y Sempronio te espulguen! ¡Oh desdichado de mí! Por ser leal padezco mal; otros se ganan por malos; yo me pierdo por bueno. El mundo es tal. Quiero irme al hilo de la gente, pues a los traidores llaman discretos, a los fieles necios. Si [yo] creyera a Celestina con sus seis docenas de años acuestas, no me maltratara Calisto. Mas esto me porná escarmiento de aquí adelante con él. Que si dijere comamos, yo también; si quiere derrocar la casa, aprobarlo; si quemar su hacienda, ir por fuego. Destruya, rompa, quiebre, dañe, dé a alcahuetas lo suyo, que mi parte me cabrá, pues dicen: a río revuelto ganancia de pescadores. ¡Nunca más perro a molino! (A, pp. 78-79)

El criado manifiesta sinceramente a los lectores/espectadores su cambio lógico de actitud. La conclusión paradójica que se desprende del monólogo podría ser formulada así: "soy leal, en mi propio perjuicio, y mi amo me juzga desleal; voy a ser desleal, en mi beneficio, y mi amo

\footnotetext{
${ }^{7}$ La primera edición data de 1499. Nuestras citas son de la edición de Alianza Editorial (Fernando de Rojas. La Celestina. Tragicomedia de Calisto y Melibea. Sexta edición. Introducción de Stephen Gilman. Notas de Dorothy S. Severin. Madrid: Alianza, 1978). De ahora en adelante, utilizaremos a menudo la letra A para referimos a esta obra. Las referencias al número de página aparecerán entre paréntesis, al final de cada cita.

${ }^{8}$ La primera edición data de 1542. Las referencias al número de página, que aparecerán entre paréntesis al final de cada cita, corresponden a la siguiente edición: Tragicomedia de Leandro y Roselia llamada Elicia, y por otro nombre cuarta obra y tercera Celestina. Madrid: Rivadeneyra, 1872. De ahora en adelante, utilizaremos a menudo la letra B para referirnos a esta obra.
} 
me juzgará leal." El parlamento de Pármeno está construido de tal manera que el personaje no tiene elección. La actitud de lealtad que había mantenido hasta entonces aparece inviable, debido al comportamiento de todos los personajes que le rodean, incluido el propio Calisto, que prefiere un criado desleal que satisfaga sus caprichos a otro leal que le sermonee, tal como había quedado patente poco antes:

2a)

CAL.-¡Palos querrá este bellaco! [...] Cuanto remedio Sempronio acarrea con sus pies, tanto apartas tú con tu lengua [...]

PARM.-Señor, flaca es la fidelidad que temor de pena la convierte en lisonja (A, p. 78)

Celestina representa una sociedad en crisis, en la cual la sumisión feudal es un valor escasamente cotizado. Según la tesis de Maravall, ${ }^{9}$ los criados han cobrado conciencia de que ya no son más que los asalariados de su amo. Ello se debe a la transformación de las relaciones sociales que se instaura con la sociedad burguesa (naciente hacia el momento de aparicion de $\mathrm{A}$ ), cuyo comportamiento ideológico ${ }^{10}$ no es ya la lealtad, sino el provecho. ${ }^{11}$ Este "aburguesamiento" implica lógicamente una cierta secularización de la visión del mundo, ${ }^{12}$ patente en este monólogo en la omisión de términos pertenecientes al campo semántico 'religión', tanto más significativa cuanto que el discurso religioso es casi omnipresente en la obra.

${ }^{9}$ José Antonio Maravall. El mundo social de «La Celestina». $2^{\text {a }}$ edición revisada y aumentada. Madrid: Gredos, 1968.

${ }^{10} \mathrm{El}$ término "comportement idéologique» aparece en Joseph Gabel, Idéologies, París: Anthropos, [1974], pp. 40-45. Se trata de un comportamiento que no tiene en cuenta las consecuencias negativas de la aplicación de cualquier ideología (es decir, que ignora lo que la ideología no muestra).

11 «L'éthique du bourgeois échangiste, qui se développe du XIII" au XV" siècle, [...] peut ainsi se résumer: la fin justifie les moyens, attendu que cette fin est le profit.» Gérard Mairet, «L'éthique marchande,» en François Chatelet (dir.), Histoire des idéologies 2. De l'Église à l'État du IX' au XVII' siècle. [París]: Hachette, 1978, p. 212.

12 «C'est l'avènement d'une culture entièrement laïcisée qui marque la mentalité du bourgeois échangiste [...]. Le passage du monde rural à une civilisation urbaine s'opérait grâce au marchand; cette transformation [...] n'a pu se faire qu'à l'intérieur d'une idéologie laïque.» Ibid., pp. 225-226. 
El cambio de actitud de Pármeno ya había sido sugerido unas páginas antes del monólogo, por medio de un aparte a Sempronio (el primero que inicia Pármeno en toda la obra), en el cual se manifiestan tanto el afán de lucro como el conflicto entre amos y criados:

3a)

PARM.- ¿Qué le dio, Sempronio?

SEMP.-Cient monedas en oro.

PARM.- ¡Hi, hi, hi!

SEMP._- ¿Habló contigo la madre?

PARM.-Calla, que sí). (A, p. 73)

El procedimiento del aparte (que siempre es implícito pues, como es bien sabido, no existen acotaciones escénicas explícitas en Celestina) cumple la función de subrayar la situación conflictiva entre amo y criados, sea ésta sintomática de su "deslealtad," como en el caso anterior, o de su no menos subversiva (para la mentalidad medieval) puesta en tela de juicio del comportamiento o las declaraciones del amo. Asi ocurre en el aparte de Sempronio al comienzo de la obra, provocado por, la declaración de Calisto de que preferiría ir al purgatorio que al paraíso («a la gloria de los santos») si su amor no se viese correspondido (si no lograse extinguir el fuego "que me quema»):

4a)

SEMP.- (Algo es lo que digo; a más ha de ir este hecho; no basta loco, sino hereje.) (A, p. 49)

En este caso, ante la insistencia de Calisto por saber lo que está murmurando el criado, Sempronio termina por desvelarle su pensamiento, aunque sólo a medias ${ }^{13}$ (omite la calificación de «loco»):

5a) CAL.- ¿No te digo que hables alto cuando hablares? ¿Qué dices? SEMP.-Digo que nunca Dios quiera tal; que es especie de herejía lo que agora dijiste.

CAL.-¿Por qué?

SEMP.-Porque lo que dices contradice la cristiana religión.

CAL.-¿Qué a mí?

${ }^{13}$ «Los personajes que advierten el murmurado aparte suelen pedir $\mathbf{u}$ ordenar que se repitan esas palabras en alta voz, y el interlocutor responde conservando alguna de las palabras que presume haberse oído." María Rosa Lida de Malkiel,

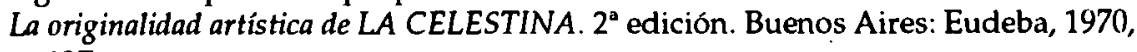
p. 137. 
SEMP.-_Tú no eres cristiano?

CAL.-_Yo? Melibeo soy y a Melibea adoro y en Melibea creo y a Melibea amo. (A, pp. 49-50)

La acusación del criado parece grave, pues la herejía, en la España de la época, suponía poco menos que un delito de lesa patria. Lo peor es que el señor parece "espontanearse"14 de manera singular. No sólo no replica a la acusación, sino que la confirma de manera escandalosa (para la época): «Melibeo soy y a Melibea adoro y en Melibea creo y a Melibea amo.» Esta declaración podría interpretarse como una parodia de la abjuración inquisitorial ${ }^{15}$ que, en lugar de negar la "herejía," la reafirma. Por si quedara alguna duda sobre la perversión del código religioso operada por Calisto, poco después, ante la acusación de su criado de que, con su actuación, se somete a una mujer (concretización por parte de Sempronio del topos misógino medieval), Calisto replica:

6a)

CAL.- ¿Mujer? ¡Oh grosero! ¡Dios, dios!

SEMP. - ¿Y así lo crees? ¿O burlas?

CAL.- ¿Que burlo? Por dios la creo, por dios la confieso y no creo que hay otro soberano en el cielo; aunque entre nosotros mora. (A, p. 51)

Si anteriormente nos referíamos a la omisión de términos religiosos en el monólogo de Pármeno, su superabundancia en los parlamentos de Calisto es, curiosamente, sintomática de un mismo estado de cosas. Pármeno obvia el discurso religioso, Calisto lo instrumentaliza para elogiar a la amada; ${ }^{16}$ es decir, con un fin no estrictamente religioso.

14 «Espontánea: la confesión que un incurso en hechos o dichos contrarios directa o indirectamente a la fe católica, hace de su propia voluntad al Santo Oficio de la Inquisición, pidiendo ser absuelto de cualesquiera censuras a que haya incurrido [...]. Espontanearse: es hacer una Espontánea» (Juan Antonio Llorente. «Explicación de las palabras y frases técnicas que se usaban en el Santo Oficio," en Historia crítica de la Inquisición en España (4 vols), Madrid: Hiperión, $1980,1,24$. El autor, antiguo inquisidor, escribió esta obra hacia los años 10-20 del siglo $X(X)$.

15 «Abjuración: es detestación de la herejía» (Ibid.).

${ }^{16}$ Lida de Malkiel utiliza el término de «hipérbole sacroprofana» para referirse a este «elogio de lo profano en términos sagrados» ( Lida de Malkiel. La originalidad artística..., p. 369). 
Se produce así la desemantización de este discurso, a causa (como en el monólogo de Pármeno) de la secularización de la visión del mundo. Curiosamente, este vaciamiento de sentido es similar al que sufre la mitología antigua en la literatura de la época (también presente en Celestina); de esta manera, la religión cristiana se convierte, como los mitos clásicos, en una mera referencia simbólico-cultural. ${ }^{17}$

Calisto no es el único personaje que instrumentaliza el discurso religioso. También Celestina lo hace para conseguir lo que se propone. Obsérvese, a título de ejemplo, cómo este personaje introduce el verdadero objeto de su visita cuando, en la primera entrevista con Melibea, ésta iba a despedirla:

7a)

MEL.-[...] ¿Y no sabes que por divina boca fue dicho, contra aquel infernal tentador, que no de sólo pan viviremos? Pues así es, tentador, que no el sólo comer mantiene. Mayormente a mí, que me suelo estar uno o dos días negociando encomiendas ajenas ayuna, salvo hacer por los buenos, morir por ellos. Esto tuve siempre, querer más trabajar sirviendo a otros, que holgar contentando a mí. Pues, si tú me das licencia, diréte la necesitada causa de mi venida, que es otra que la que hasta agora has oído y tal, que todos perderiamos en me tornar en balde sin que la sepas. (A, p. 93)

Bien sabemos cuál es esta "necesitada causa.» El fin último de Celestina no es, sin embargo, éste, sino el de conseguir, gracias a sus buenos servicios, el mayor beneficio económico posible. Un mismo motivo le impulsaba en su juventud a ejercer de prostituta, como bien sabe el lector/espectador desde que la describiera Pármeno, en el primer auto:

${ }^{17}$ «Si en Celestina, conviven, codo a codo, los mitos clásicos con la teología cristiana, $[\ldots]$ la única solución viable $[\ldots]$ es reducir ambos sistemas a puras referencias simbólico-culturales. Esta solución, que en rigor no es ni cristiana ni anticristiana, es probablemente el recurso normal de muchos escritores de la época, aunque es de ley, en estos casos, que se marque textualmente la jerarquía entre los dos sistemas. [...] Rojas $[\ldots]$ se olvidó de dejar en su texto el "constat" de la supremacía de la teología cristiana sobre los mitos antiguos.» Félix Carrasco, "La recepción del planto de Pleberio en las traducciones e imitaciones de Celestina" (Ponencia presentada en el III Congreso Internacional de la Asociación de Literatura Hispánica Medieval celebrado en Lisboa en 1991, cuyas actas van a ser publicadas en breve). 
8a)

PARM.-[...] de esto ["puta vieja alcoholada»] es nombrada y por tal título conocida. Si entre cient mujeres va y alguno dice: «iPuta vieja!", sin ningún empacho luego vuelve la cabeza y responde con alegre cara. En los convites, en las fiestas, en las bodas, en las cofadrías, en los mortuorios, en todos los ayuntamientos de gentes, con ella pasan tiempo. Si pasa por los perros, aquello suena su ladrido; si está cerca las aves, otra cosa no cantan; si cerca los ganados, balando lo pregonan; si cerca las bestias, rebuznando dicen: «iPuta vieja!»; las ranas de los charcos otra cosa no suelen mentar. Si va entre los herradores, caldereros, arcadores, todo oficio de instrumento forma en el aire su nombre. Cántanla los carpinteros, péinanla los peinadores, tejedores; labradores en las huertas, en las aradas, en las viñas, en las segadas con ella pasan el afán cotidiano [...]. (A, pp. 59-60)

Esta hiperbólica etopeya-pese al rebajamiento del personaje-es sintomática de un hecho fundamental para la comprensión de la obra: Celestina, personaje bien conocido por todos (salvo por Calisto; verosimilitud obliga), es única (reconocible «entre cient mujeres»). Unica y fundamental, razón por la cual este personaje había sido evocado por Pármeno en el monólogo con que se inicia este estudio, pues Celestina es quien abre los ojos a Pármeno sobre el verdadero sentido de su posición: está en la casa de Calixto, pero no es de ella; no es más que un mercenario para su amo. No es un "natural," es un extraño, como lo ha sido en otras partes [...]. Los criados conservan siempre [...] una clara noción de la maldad de Celestina [...]. Pero no pueden encontrar, en el estado social en que se hallan colocados, motivos para abstenerse. Todos $[\ldots]$ no tienen más que un objetivo: aprovecharse. ${ }^{18}$

Lida de Malkiel, que había acertado plenamente al señalar la aguda conciencia profesional de Celestina, ${ }^{19}$ no se muestra, a nuestro juicio, tan fina al considerar que el hecho de «trata[r] de sacar el mayor partido posible de sus servicios" sea «inherente a su codicia senil.. ${ }^{20} \mathrm{~A}$ nuestro entender, Celestina no hace sino respetar una regla básica de «l'éthique marchande, [qui] consiste à moraliser le profit.»" No por otra

${ }^{18}$ Maravall. El mundo social..., p. 89.

${ }^{19}$ Cf. Lida de Malkiel. La originalidad artística..., pp. 515-519.

${ }^{20}$ Ibid., p. 516.

${ }^{21}$ Mairet, “L'éthique marchande," p. 212. 
razón precia este personaje «a tal punto [...] su oficio que en su cumplimiento muestra las únicas notas morales positivas..22

Dos visiones del mundo diametralmente opuestas se enfrentan en Celestina. El texto muestra la inviabilidad de un modeio de sociedad ya decadente (el feudal), ostensible en la desemantización del discurso religioso, y en el hecho de que los personajes (tanto el "alto" como el "bajo") actúen movidos por su propio provecho, acatando así una de las reglas básicas de la concepción burguesa de la sociedad. Es en el personaje de Celestina (después de todo, una comerciante) donde la lógica burguesa se manifiesta más nítidamente, ${ }^{23}$ pues el comportamiento ideológico del provecho $^{24}$ se cristaliza en la instrumentalización del discurso religioso, síntoma, a su vez, de la emergencia de esta ideología burguesa.

Sin embargo, el lector/espectador sabe que la búsqueda del provecho va a ser fatal para todos los personajes. El final trágico (entre otros elementos) muestra las consecuencias negativas de este comportamiento ideológico. Celestina se distancia así, también, de la ideología emergente.

Una vez llegados a esta conclusión, pudiera parecer inútil la comparación entre Celestina y la Elicia, pues en esta obra, a diferencia de lo que ocurre en otras imitaciones, «el componente trágico es restablecido

\footnotetext{
${ }^{22}$ Lida de Malkiel. La originalidad artística..., p. 515.
}

23 La mentalidad burguesa en formación de Celestina no le impide, sin embargo, contagiarse de un elemento del discurso señorial, como es la honra. No pretendemos desarrollar aquí un análisis exhaustivo sobre el empleo de la honra en Celestina, lo cual requeriría un trabajo de mayor envergadura. Limitémonos a señalar que, como "dans le Quichotte, [...] nous ne retrouvons pas seulement (intégrés de manière contradictoire) des discours appartenant aux deux systèmes «sociaux» conflictuels qui coexistent en collision intersystémique [...]. Du fait même du caractère peu pacifique d'une telle coexistence, nous retrouvons aussi des contaminations interdiscursives constantes." Antonio Gómez-Moriana, "Pragmatique du discours et réciprocité de perspectives," en Catherine Poupeney Hart y Antonio Gómez-Moriana (eds.), Parole exclusive, parole exclue, parole transgressive. Marginalisation et marginalité dans les pratiques discursizes, Longueuil: Préambule, 1990, p. 29.

24 Véanse notas 10 y 11 
e incluso incrementado.» ${ }^{25}$ Por si esto no fuera suficiente, también el provecho es el catalizador de la acción, tal como se manifiesta en el monólogo de Oligides, cuya filiación con el de Pármeno no ofrece lugar a dudas:

$1 b)^{26}$

Olig. [...] ¿qué se me da á mí? Ahórquenlo en buen día claro, siquiera se muera ó le tome el diablo. Andaos por ahí a decir verdades y moriréis por los hospitales; no es tiempo de eso, ya me llamaba sancto, y pardios las buenas doctrinas de Eubulo, criado antiguo de esta casa, me habían casi convertido; pero poco puedo medrar con sus devociones y sanctidades; no ando yo tras eso, ni es esto lo que busco. Quiero pesquisar y inquerir con mi pensamiento la entrada á Roselia y ser alcahuete, venga el bien y venga por do quisiere, á tuerto o á derecho nuestra casa fasta el techo, que buena parte me cabrá de sus amores, que á rio vuelto, como dicen, ganancia de pescadores. (B, p. 12)

La estructura narratológica que se desprende de los monólogos de Pármeno y de Oligides ofrece rasgos evidentes de similitud. El criado también se encuentra ante la disyuntiva de seguir siendo leal a su amo o dejar de serlo, y en ambos casos adopta la segunda alternativa. Sin embargo, a diferencia de Pármeno, Oligides sí tiene posibilidad de elección: Eubulo, el personaje evocado, sostiene la opción contraria, no sólo presentada como posible, sino, sobre todo, como la única correcta.

El hecho de que los monologantes de $\mathrm{A}$ y $\mathrm{B}$ evoquen a otro personaje nos parece fundamental para el análisis de su producción de sentido. Poco importa si la caracterización del personaje evocado es positiva o negativa, si su opinión va a servir de norma de conducta o no a los otros personajes. El efecto producido en el lector/espectador es, en cierto sentido, el mismo: el de (comenzar a) percibir la importancia del personaje evocado en el desarrollo de la acción. Obsérvese la diferencia de función de uno y otro personajes: Eubulo es el arquetipo del criado leal, Celestina es el personaje instigador de la deslealtad al amo; la amoral-si no inmoral-Celestina instrumentaliza el discurso cristiano en beneficio propio, Eubulo-baluarte de moral —es el perfecto vehiculador

${ }^{25}$ Carrasco («La recepción...»).

${ }^{26} \mathrm{El}$ código que precede cada cita remite al fragmento de A (como, en este caso, $1 \mathrm{~b}$-monólogo de Oligides - es el correlato de 1a-monólogo de Pármeno). Para la perfecta comprensión de este trabajo, recomendamos la confrontación entre ambos fragmentos, así como entre sus análisis respectivos. 
de este discurso. Resultado: en el monólogo de $B$, al contrario de lo que ocurría en su paralelo de $\mathrm{A}$, abundan los términos que refieren al campo semántico 'religión', de manera clara ("sancto," "convertido," «devociones," "sanctidades») o un tanto solapada («diablo,» "pardios," «buenas doctrinas»). ${ }^{27}$

A pesar de su voluntad manifiesta de entroncar la Elicia con Celestina, Sancho de Muñón no se limita a calcar fielmente el modelo. Si así fuese, Oligides debería comportarse en toda ocasión como una especie de sustituto de Pármeno, y Eubulo sería, por lo tanto, un nuevo Sempronio. Este paralelismo no se respeta siempre, pues también Eubulo cita textualmente a Pármeno:

2b)

Eub. Flaca es la fidelidad, como decia Parmeno, que temor de pena la convierte en lisonja; nunca por sus amenazas [de Lisandro] dexaré de decir la verdad. (B, p. 29)

La primera parte del parlamento (salvo, claro está, la apostilla, referencia metatextual que adscribe la obra en el "ciclo celestinesco") reproduce con exactitud las palabras de Pármeno; la segunda parte es responsabilidad de Eubulo. Es lógico que así sea, pues se trata de su autocaracterización. En ella se adelanta el hecho de que este personaje va a ser mucho más inflexible en sus opiniones que su supuesto modelo: a diferencia de Pármeno, Eubulo será leal a su amo hasta el final, aun a pesar de éste. Sus parlamentos así lo certifican, esté Lisandro ausente de escena o no. En este último caso, Eubulo utiliza en algunas ocasiones el aparte, con lo cual se subraya el poco efecto que sus palabras provocan en Lisandro:

3b)

Eub. Escocióle el buen consejo.

Lis. ¿Qué dices?

$E u b$. Digo que voy. (B, p. 15)

También Oligides recurre al aparte en numerosas ocasiones. Especialmente significativo es el siguiente, por sus reminiscencias con el original:

2La parcial desemantización de estas palabras (especialmente "pardios") es obra del desgaste por el uso; en ningún caso, de su consciente instrumentalización en un discurso ajeno. 
4b)

Lis. [...] ¿Hablaste con aquella que par no tiene en la tierra, y en el cielo compete con los bienaventurados?

Olig. Otro Calixto hereje tenemos.

Lis. ¿Qué dices de Calixto?

Olig. Que no tuvo tanta razón para amar á Melibéa, aunque fué mucha, como tú tienes para querer y desear á Roselia. (B, p. 17)

De nuevo, nos encontramos ante una referencia metatextual que suaviza la (presumiblemente) grave acusación del criado. A Lisandro le falta el carácter corrosivo de las declaraciones de Calisto, como lo testimonia la diferencia de tono perceptible en los enunciados de los dos enamorados: mientras que Calisto situaba el goce terreno por encima del celestial (prefería ir al purgatorio que al paraíso si su amor no se veía correspondido), Lisandro ofrece una descripción de la amada puramente convencional, que se enmarca en la tradición cancioneril. Melibea, que ocupaba en el corazón de Calisto el lugar de Dios mismo (que era Dios; véase 6a), es reemplazada en $B$ por Roselia, personaje que, mucho más modestamente, no pasa de ser un ángel:

$5 b, 6 b)$

Lis. Por ángel tengo y juzgo, y ansí la confieso, aquella cuyo amor hace que ame á Dios como causa de tal efecto.

Olig. Perviertes el órden, señor.

Lis. ¿En qué manera?

Olig. Porque todo lo criado en razon del Hacedor amar se debe, tú al reves haces y lo contrario sigues de lo que la maestra natura nos enseña, que es amar al principio por sí mesmo, y la labor en su orígen.

Lis. ¿San Pablo no dice que de lo visible venimos en conocimiento de lo invisible? (B, pp. 6-7)

El conflicto es aquí banal. La declaración inocente del señor (como ha sido señalado más arriba, la comparación ángel = amada se ajustaba perfectamente a las expectativas de la retórica amorosa del momento) provoca la acusación por parte del criado no de herejía, sino del pecado venial (o, en todo caso, menos grave) de "pervertir el orden." Consecuentemente, la cita irrespetuosa de 5 a se vuelve respetuosa en $5 \mathrm{~b}$ : Lisandro, si bien no cambia de opinión, la defiende dentro de los cánones impuestos por el código retórico de la época (la argumentación por cita de autoridad; y de una autoridad, nunca mejor dicho, "canónica": San Pablo). Es decir, utilizando el código de manera "normal" (sujetándose a la norma), no transgresora. De ahí que su amor por Roselia no 
implique negar a Dios; antes al contrario, reafirma su autoridad («aquella cuyo amor hace que ame á Dios»).

Lisandro, por lo tanto, no instrumentaliza el discurso religioso (o, en todo caso, lo hace mínimamente, sin pervertirlo). Otro es el caso de Celestina, en este sentido poco diferente al original. ${ }^{28}$ Es comprensible, pues la alcahueta de la Elicia no es otra que la propia Elicia de A, y como tal, es previsible que haya gozado de las sabias enseñanzas de su tía:

$\left.8 b^{\prime}\right)$

$E u b .[\ldots]$ ¿quién es esta negra señora que venimos á traer de la mano?

Olig. Yo te lo diré; bien habrás oído mentar á Celestina la barbuda, la que tenía el Dios os salve por las narices, aquella que vivía á las tenerías; ¿no caes?

Eub. ¡Oh! joh! di, di, que ya caigo, que como ha habido tantas y hay, no sabía por quién decias.

Olig. Ésta dexó dos sobrinas, Areusa y Elicia [...]; quedó Elicia ya vieja y de dias, la cual viendo que los años arrugaban su rostro, y que su casa no se frecuentaba como solia de galanes, ni ménos sus amigos la visitaban, determinó, pues con su cuerpo no podia ganar de comer, ganallo con el pico y tomar el oficio de su tia. (B, p. 32)

Este fragmento, como tantos otros ya señalados con anterioridad, contiene marcas metatextuales que tienen por función «conectar con el original, que es uno de los invariantes del género celestinesco.» ${ }^{29} \mathrm{La}$ proposición de relativo «aquella que vivía á las tenerías,» que ya sirviera

${ }^{28}$ Baste un ejemplo de la instrumentalización del discurso religioso por parte de esta nueva Celestina, en circunstancias similares a las de $7 \mathrm{a}$ :

7b)

Cel. ¡Ay señora! y ¿qué mal es, ó qué pecado, si con mi vista y palabra puedo dar la vida al doliente, consentir que me vea y hable? $Y$ áun es obra de perfeccion dar industria y forma para ello.

Ros. Si no es más de eso, cosa sancta y buena es.

Cel. ¿Y cómo sancta? Lo contrario hacer sería pecado mortal, porque cualquier que puede á otro salvar la vida sin pecado ó notable peligro suyo, y no lo hace, peca; cuanto más que entre las obras de misericordia es dado consejo que visitemos los enfermos. (B, pp. 98-99)

${ }^{29}$ Carrasco. "La recepción...». 
de referencia identificadora en A, reaparece en B para indicar que el personaje evocado no es otro que la Celestina original. Sin embargo, es curioso que a esta referencia le precedan otras («la barbuda," «la que tenía el Dios os salve por las narices"), también extraidas del original, pero que nunca habían aparecido juntas en éste. Esta abundancia de referencias identificadoras sugiere que el personaje no es ya tan identificable como lo era en A. La respuesta de Eubulo confirma esta sospecha ("como ha habido tantas y hay...").

La "inflación" de medianeras es un tema recurrente a lo largo de toda la obra. He aquí otro ejemplo:

Olig. [...] al fin mal se tañe la vihuela sin tercera; en el cielo sin medianera no se alcanza cosa que buena sea, cuanto más en el suelo, lo demas es andar de mula coxa.

Lis. ¿Conoces tú alguna?

Olig. No una, sino ciento; está sembrada la ciudad de ellas, no hay mujer cantonera que no tenga su vieja al lado para que sea corredora de estas ventas y compras [...]. (B, p. 30, mi subrayado)

Aquella mujer reconocible "entre cient mujeres» (véase 8a) ya no es más que una entre "ciento.» Del personaje único de A se pasa a la multiplicación de celestinas en $B$; de su notoriedad, al anonimato.

Intentaremos explicar brevemente el motivo de esta transformación. La alcahueta es un antiguo personaje literario/teatral presente ya en las comedias griega y latina que, en virtud del "decoro" aristotélico, nunca había gozado de un puesto protagónico antes de la obra de Rojas. En este sentido, la Tragicomedia de Calisto y Melibea supuso una gran ruptura con el sistema literario tradicional, como lo atestigua el hecho de que la obra pronto fuese conocida con el nombre de Celestina, en desmedro de los personajes "altos" Calisto y Melibea. En Elicia, al "multiplicar" las celestinas, al hacerlas difícilmente distinguibles unas de otras, lo que se produce es la reinstalación del personaje en el lugar secundario que se le tenía asignado desde antiguo. ${ }^{30}$

${ }^{30}$ Este rebajamiento no es, por cierto, privativo de esta imitación de la obra de Rojas. Incluso hay «un reducido número [de imitaciones que] elimina el personaje [de Celestina] y deja la tercería a cargo de los criados." Lida de Malkiel. La originalidad artística..., p. 572. 
No es ésta la única ocasión en que un elemento transgresor de A es desactivado en $B$ para reincorporarlo a la tradición: recuérdese, por ejemplo, cómo las herejes declaraciones de Calisto (comparación amada=Dios) se reinscriben, por boca de Lisandro, en la convención cancioneril de la época (amada=ángel). De esta manera, el discurso religioso, que había sufrido un proceso de instrumentalización en $A$, "vuelve a la normalidad" en B, acabando por resemantizarse mediante el recurso a la cita de autoridad.

En cuanto al provecho económico (elemento fundamental para la comprensión de Celestina), es también el motor de la actuación de la mayor parte de los persónajes de la Elicia. Pero no de todos ellos: Eubulo (personaje "positivo," portador de valores opuestos a los burgueses) no cambiará de posición en toda la obra. Su filiación ideológica con "el primer" Pármeno (el que aún es leal a su amo, antes del monólogo) es evidente. ${ }^{31}$ De esta manera, otro elemento especialmente conflictivo para la mentalidad hegemónica de la época es desaciivado. Todo lo anterior indica que, mientras que Celestina se mantenía distanciada tanto de la visión burguesa ascendente como de la medieval descendente, la Tragicomedia de Lisandro y Roselia opta claramente por esta última. O, mejor dicho: opta por la versión remozada de esta última, que pronto aflorará oficialmente con el nombre de Contrarreforma. ${ }^{32}$

${ }^{31}$ A este respecto, es especialmente significativo que, por lo que respecta a los criados, la mayor parte de las marcas intertextuales que hay en B (poco importa si aparecen en Oligides o en Eubulo) no apuntan sino a uno de los criados de A: Pármeno. Y al Pármeno, en la mayoría de los casos, de antes de la "conversión". Obsérvese la clara marca del "primer Pármeno" en Oligides:

PARM.-Señor, más quiero que airado me reprehendas, porque te doy enojo, que arrepentido me condenes, porque no te di consejo [...]. (A, p. 77)

Olig. Mi deber hago, que es darte consejo porque no me condenes arrepentido. 2b).

Para las reminiscencias del "primer Pármeno" en Eubulo, cotéjese 2a) con

${ }^{32}$ El Concilio de Trento comienza tres años después de publicada la Elicia, en 1545. «Se crea un distanciamiento radical frente a la actitud de Rojas respecto a los problemas ideológicos debatidos [...]; es evidente que el medio siglo que separa las dos obras marca una trayectoria desde la apertura hacia el cierre en cuanto a la circulación de ideas." (Carrasco, "Notas...," p. 47). Esta cita se refiere a otra obra del ciclo celestinesco aparecida hacia la misma época (la Comedia de Sepúlveda), pero bien se puede aplicar a la Elicia. 
La Elicia (como cualquier otra obra del "ciclo celestinesco") no deja de ser una reproducción parcial del modelo. Parcial en el doble sentido de la palabra: no totalmente idéntica al original y sintomática de una parcialidad (ideológica, axiológica) no necesariamente coincidente con la de Celestina. Los "desvíos" del modelo, desde esta óptica, deben ser entendidos como un «intento de rectificar la escritura de Rojas, conciliándola con los valores de la sociedad española de la época. $\${ }^{33} \mathrm{Del}$ éxito de operaciones de este tipo dependerá la capacidad del sistema para neutralizar y asimilar las voces discordantes que él mismo produce.

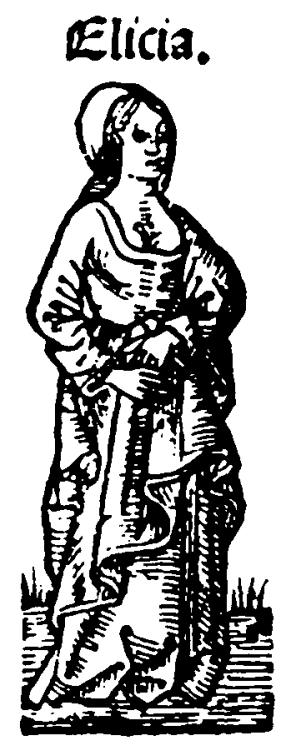

Sevilla: J. Cromberger, 1525

${ }^{33}$ Ibid., p. 46. 